\section{Conhecimento sobre fatores de risco para doenças crônicas: estudo de base populacional}

\author{
Knowledge on risk factors for chronic diseases: \\ a population-based study
}

\footnotetext{
${ }^{1}$ Escola Superior de Educação Física Universidade Federal de Pelotas, Pelotas, Brasil. 2 Faculdades Atlântico Sul, Pelotas, Brasil.

3 Programa de Pós-graduação, em Epidemiologia, Universidade Federal de Pelotas, Pelotas, Brasil.

Correspondência T. T. Borges

Escola Superior de Educação Física, Universidade Federal de Pelotas.

Rua Luiz de Camões 625, Pelotas, RS

96055-630, Brasil.

thiagotborges@yahoo.com.br
}

\begin{abstract}
The aim of the present study was to evaluate public awareness of the association between four behavioral factors (sedentary lifestyle, smoking, alcohol abuse, and inadequate diet) and eight diseases (diabetes, hypertension, AIDS, osteoporosis, lung cancer, depression, liver cirrhosis, and acute myocardial infarction). We conducted a population-based cross-sectional study including 2,096 individuals 10 years or older. A random clustered sampling strategy was used. For each behavioral factor, a knowledge score was constructed, ranging from zero to eight points. The highest mean score was observed for inadequate diet (5.3), followed by smoking (5.1), sedentary lifestyle (4.7), and alcohol abuse (4.5). Overall, higher knowledge scores were observed among people with high socioeconomic status and more schooling, and in intermediate age groups. Government health promotion strategies are needed to raise public awareness of risk factors for chronic diseases.
\end{abstract}

Chronic Disease; Behavior; Knowledge
Thiago Terra Borges 1,2 Airton José Rombaldi 1 Alan Goularte Knuth ${ }^{3}$ Pedro C. Hallal 1,3

\section{Introdução}

Um dos aspectos importantes para a melhoria da qualidade de vida de uma população é o aumento da sua capacidade de compreender os fenômenos relacionados à sua saúde. $\mathrm{O}$ conhecimento sobre um determinado desfecho em saúde pode ser útil para ajudar a evitar o surgimento de um agravo, podendo também influenciar na busca pelo tratamento, quando a doença já está estabelecida. Para tanto, espaços de uso e fluxo populacional, como escolas, universidades, veículos de comunicação e serviços de saúde, são potenciais difusores dessa informação, ainda que a forma de impactar perante a população geral possa ser distinta 1 .

No Brasil, a produção científica referente ao conhecimento populacional sobre indicadores de saúde ainda é limitada, visto que poucas pesquisas de base populacional avaliaram esse tema 2 . Não há registros na literatura de estudos brasileiros com o propósito de avaliar conjuntamente o quanto a população conhece sobre fatores de risco, como tabagismo, sedentarismo, alimentação inadequada e consumo excessivo de álcool, às doenças e agravos não transmissíveis. É possível que diferenças culturais, sócio-econômicas e regionais estejam associadas com os níveis de informação. Maiores graus de escolaridade e acesso aos veículos de informação podem afetar o conhecimento e/ou a capacidade de compreensão das informações recebidas 3 . 
O presente estudo avalia o conhecimento populacional sobre as associações entre quatro fatores comportamentais (sedentarismo, tabagismo, consumo excessivo de álcool e alimentação inadequada) e oito morbidades (diabetes, hipertensão arterial, AIDS, osteoporose, câncer de pulmão, depressão, cirrose hepática e infarto agudo do miocárdio).

\section{Métodos}

Foi realizado um estudo transversal de base populacional no final do ano de 2007 em Pelotas, Rio Grande do Sul, Brasil. A cidade possui aproximadamente 340 mil habitantes. O processo de amostragem foi probabilístico por conglomerados, realizado em dois estágios. As unidades amostrais primárias foram os setores censitários delimitados pelo Instituto Brasileiro de Geografia e Estatística (IBGE. Censo demográfico, 2000. http://www.ibge.gov.br, acessado em Fev/2008) em 2000. Inicialmente, foi feita uma listagem de todos os setores censitários domiciliares da cidade $(n=404)$; posteriormente, sortearam-se aleatoriamente aqueles que foram incluídos na amostra $(n=45)$. Em cada setor, foi identificado o ponto de partida do estudo, a partir do qual, a cada cinco domicílios, foram selecionadas sistematicamente as casas visitadas (20 por setor). Foram incluídos na amostra todos os indivíduos com idade igual ou superior a dez anos residentes nos domicílios sorteados.

O questionário utilizado foi pré-testado em setor censitário não sorteado para a amostra final do estudo. Tendo em vista a inexistência de instrumentos validados para a avaliação de conhecimento sobre fatores de risco para doenças e agravos não transmissíveis, optou-se por utilizar um instrumento criado pelos pesquisadores (Tabela 1).

O instrumento (Tabela 1) avalia o conhecimento populacional sobre a influência de quatro fatores de risco (sedentarismo, tabagismo, consumo excessivo de álcool e alimentação inadequada) sobre doenças e agravos não transmissíveis e oito morbidades (diabetes, hipertensão arterial, AIDS, osteoporose, câncer de pulmão, depressão, cirrose hepática e infarto agudo do miocárdio). A definição de certo ou errado para cada resposta baseou-se em artigos de revisão de literatura sobre a associação de cada fator de risco e doença ou agravo, com preferência para revisões sistemáticas e meta-análises. A Tabela 2 mostra o que foi considerado certo para cada associação investigada, conforme referência bibliográfica que embasou tal conclusão. Para cada um dos quatro fatores, foi construído um escore de acertos, que poderia variar de zero a oito pontos.

As variáveis independentes analisadas foram sexo, idade (categorizada em décadas), cor da pele (observada pelo entrevistador), escolaridade (em anos completos de estudo) e nível sócio-econômico (dividido em quatro categorias, tendo por base o Critério de Classificação Econômica da Associação Brasileira de Empresas de Pesquisa - ABEP. http://www.abep.org, acessado em Ago/2008).

As entrevistas foram realizadas por mulheres que passaram por um processo de treinamento com duração de 40 horas. Um total de $10 \%$ da amostra foi revisitado para fins de controle de qualidade. Os dados obtidos pelo questionário foram duplamente digitados no programa Epi Info 6.0 (Centers for Disease Control and Prevention, Atlanta, Estados Unidos), e as análises estatísticas foram realizadas no pacote estatístico Stata 9.1 (Stata Corp., College Station, Estados Unidos).

A análise de dados incluiu uma descrição da amostra, estratificada por sexo, com cálculo de proporções para cada subgrupo de exposição. Uma análise descritiva das respostas corretas de cada fator de risco relacionado às doenças e agravos não transmissíveis foi conduzida. Em seguida, com o intuito de se verificar se os conhecimentos sobre cada um dos quatro fatores de risco para doenças e agravos não transmissíveis estavam fortemente correlacionados ou eram independentes, calculou-se a correlação de Spearman entre os escores obtidos. Por fim, foram comparadas as médias dos escores entre os subgrupos das variáveis independentes. A significância foi avaliada pelos testes $t$, análise de variância (ANOVA) e Kruskal-Wallis, conforme o número de grupos sendo comparados, respeitando-se os pressupostos de cada teste. Foi realizada análise ajustada por regressão linear múltipla. Tendo em vista a distribuição dos escores de conhecimento, testou-se também o uso de regressão de Poisson, mas, como os resultados foram consistentes, optou-se por apresentar apenas os obtidos pela regressão linear múltipla. O processo amostral por conglomerados foi considerado nas análises, por meio do conjunto de comandos "svy" do Stata 9.1. O ponto de corte para significância estatística foi definido em $5 \%$.

O projeto foi aprovado pelo Comitê de Ética em Pesquisa da Escola Superior de Educação Física da Universidade Federal de Pelotas. As entrevistas somente foram realizadas após consentimento dos entrevistados. 
Questionário utilizado no estudo.

\begin{tabular}{|c|c|c|c|c|}
\hline \multicolumn{5}{|c|}{ Você acha que a falta de atividade física, sedentarismo, pode causar: } \\
\hline Diabetes mellitus, açúcar alto no sangue? & (0) Não & (1) $\operatorname{Sim}$ & (2) Desconhece a doença & (9) IGN \\
\hline Pressão alta? & (0) Não & (1) $\operatorname{Sim}$ & (2) Desconhece a doença & (9) IGN \\
\hline AIDS? & (0) Não & (1) $\mathrm{Sim}$ & (2) Desconhece a doença & (9) IGN \\
\hline Osteoporose, fraqueza nos ossos? & (0) Não & (1) Sim & (2) Desconhece a doença & (9) IGN \\
\hline Câncer de pulmão? & (0) Não & (1) $\operatorname{Sim}$ & (2) Desconhece a doença & (9) IGN \\
\hline Depressão? & (0) Não & (1) Sim & (2) Desconhece a doença & (9) IGN \\
\hline Cirrose, doença no fígado? & (0) Não & (1) $\operatorname{Sim}$ & (2) Desconhece a doença & (9) IGN \\
\hline Infarto do coração? & (0) Não & (1) $\operatorname{Sim}$ & (2) Desconhece a doença & (9) IGN \\
\hline \multicolumn{5}{|l|}{ Você acha que o fumo pode causar: } \\
\hline Diabetes mellitus, açúcar alto no sangue? & (0) Não & (1) $\operatorname{Sim}$ & (2) Desconhece a doença & (9) IGN \\
\hline Pressão alta? & (0) Não & (1) $\operatorname{Sim}$ & (2) Desconhece a doença & (9) IGN \\
\hline AIDS? & (0) Não & (1) $\operatorname{Sim}$ & (2) Desconhece a doença & (9) IGN \\
\hline Osteoporose, fraqueza nos ossos? & (0) Não & (1) $\operatorname{Sim}$ & (2) Desconhece a doença & (9) IGN \\
\hline Câncer de pulmão? & (0) Não & (1) Sim & (2) Desconhece a doença & (9) IGN \\
\hline Depressão? & (0) Não & (1) $\mathrm{Sim}$ & (2) Desconhece a doença & (9) IGN \\
\hline Cirrose, doença no fígado? & (0) Não & (1) $\mathrm{Sim}$ & (2) Desconhece a doença & (9) IGN \\
\hline Infarto do coração? & (0) Não & (1) $\mathrm{Sim}$ & (2) Desconhece a doença & (9) IGN \\
\hline \multicolumn{5}{|c|}{ Você acha que o consumo excessivo de bebidas alcoólicas pode causar: } \\
\hline Diabetes mellitus, açúcar alto no sangue? & (0) Não & (1) Sim & (2) Desconhece a doença & (9) IGN \\
\hline Pressão alta? & (0) Não & (1) $\mathrm{Sim}$ & (2) Desconhece a doença & (9) IGN \\
\hline AIDS? & (0) Não & (1) $\mathrm{Sim}$ & (2) Desconhece a doença & (9) IGN \\
\hline Osteoporose, fraqueza nos ossos? & (0) Não & (1) $\mathrm{Sim}$ & (2) Desconhece a doença & (9) IGN \\
\hline Câncer de pulmão? & (0) Não & (1) $\mathrm{Sim}$ & (2) Desconhece a doença & (9) IGN \\
\hline Depressão? & (0) Não & (1) Sim & (2) Desconhece a doença & (9) IGN \\
\hline Cirrose, doença no fígado? & (0) Não & (1) Sim & (2) Desconhece a doença & (9) IGN \\
\hline Infarto do coração? & (0) Não & (1) Sim & (2) Desconhece a doença & (9) IGN \\
\hline \multicolumn{5}{|c|}{ Você acha que a alimentação inadequada pode causar: } \\
\hline Diabetes mellitus, açúcar alto no sangue? & (0) Não & (1) $\mathrm{Sim}$ & (2) Desconhece a doença & (9) IGN \\
\hline Pressão alta? & (0) Não & (1) Sim & (2) Desconhece a doença & (9) IGN \\
\hline AIDS? & (0) Não & (1) $\mathrm{Sim}$ & (2) Desconhece a doença & (9) IGN \\
\hline Osteoporose, fraqueza nos ossos? & (0) Não & (1) $\mathrm{Sim}$ & (2) Desconhece a doença & (9) IGN \\
\hline Câncer de pulmão? & (0) Não & (1) $\mathrm{Sim}$ & (2) Desconhece a doença & (9) IGN \\
\hline Depressão? & (0) Não & (1) $\mathrm{Sim}$ & (2) Desconhece a doença & (9) IGN \\
\hline Cirrose, doença no fígado? & (0) Não & (1) $\mathrm{Sim}$ & (2) Desconhece a doença & (9) IGN \\
\hline Infarto do coração? & (0) Não & (1) $\mathrm{Sim}$ & (2) Desconhece a doença & (9) IGN \\
\hline
\end{tabular}

IGN: ignorado.

\section{Resultados}

A amostra incluiu 2.096 pessoas com dez anos ou mais de idade; o percentual de perdas e recusas foi de $9 \%$. A amostra incluiu $54 \%$ de mulheres, sendo a média de idade geral de 38,9 (desviopadrão - DP = 19,1) anos. Mais de $80 \%$ dos indivíduos foram classificados pelos entrevistado- res como tendo cor da pele branca. Houve um pequeno percentual de indivíduos no nível sócio-econômico E (mais pobres), os quais foram agrupados aos de classe D, totalizando $12,6 \%$. A ausência de escolaridade foi bem mais freqüente nas mulheres (6\%) em comparação aos homens (2,2\%). A variável com maior número de valores ignorados foi o nível sócio-econômico, com 33 


\begin{tabular}{|c|c|c|c|c|}
\hline Morbidade & Sedentarismo & Tabagismo & $\begin{array}{c}\text { Consumo abusivo } \\
\text { de álcool }\end{array}$ & $\begin{array}{l}\text { Alimentação } \\
\text { inadequada }\end{array}$ \\
\hline Diabetes & $\operatorname{Sim} 15$ & $\operatorname{Sim} 21$ & $\operatorname{Sim} 26$ & $\operatorname{Sim} 34$ \\
\hline Hipertensão & $\operatorname{Sim} 16$ & $\operatorname{Sim} 22$ & $\operatorname{Sim} 27$ & $\operatorname{Sim} 35$ \\
\hline AIDS & Não & Não & $\operatorname{Sim} 28$ & Não \\
\hline Osteoporose & Sim 17 & $\operatorname{Sim} 23$ & $\operatorname{Sim} 29$ & $\operatorname{Sim} 36$ \\
\hline Câncer de pulmão & $\operatorname{Sim} 18$ & $\operatorname{Sim} 24$ & $\operatorname{Sim} 30$ & $\operatorname{Sim} 37$ \\
\hline Depressão & $\operatorname{Sim} 19$ & Não & Sim 31 & $\operatorname{Sim} 38$ \\
\hline Cirrose & Não & Não & $\operatorname{Sim} 32$ & Não \\
\hline Infarto agudo do miocárdio & $\operatorname{Sim} 20$ & $\operatorname{Sim} 25$ & $\operatorname{Sim} 33$ & $\operatorname{Sim} 39$ \\
\hline
\end{tabular}

Nota: a numeração ao lado de cada "sim" é referente à literatura selecionada.

ausências de dados. A Tabela 3 descreve a amostra geral e estratificada por sexo conforme as variáveis sócio-demográficas.

A Tabela 4 apresenta a proporção de respostas corretas para cada fator de risco em relação às doenças e agravos não transmissíveis. Para o sedentarismo, a maioria dos indivíduos soube identificar as doenças não associadas a esse comportamento: 90,5\% e 81,5\% para AIDS e cirrose hepática, respectivamente. Nas doenças associadas ao sedentarismo, houve mais de $50 \%$ de acertos em todas, exceto câncer de pulmão, para a qual apenas $16,7 \%$ dos entrevistados relataram existir associação com sedentarismo.

A grande maioria (97\%) dos entrevistados respondeu corretamente que o tabagismo está relacionado com o risco de câncer de pulmão, e quase $90 \%$ mencionaram a associação existente com infarto agudo do miocárdio. Aproximadamente $90 \%$ dos sujeitos relataram corretamente não haver associação causal entre tabagismo e risco de AIDS (Tabela 4).

A difundida associação entre álcool e cirrose hepática foi identificada por $92,4 \%$ dos entrevistados. Cerca de $80 \%$ dos respondentes mencionou corretamente a existência de associação entre consumo abusivo de álcool e hipertensão arterial ou infarto agudo do miocárdio. Apenas $5 \%$ dos indivíduos afirmaram haver associação entre consumo excessivo de álcool e AIDS. Menos de 50\% conheciam a associação entre álcool e diabetes, câncer de pulmão e osteoporose (Tabela 4)

Para a alimentação inadequada, o menor percentual de acertos foi observado para a associação com câncer de pulmão $(24,4 \%)$, e o maior para a ausência de associação com AIDS (90,8\%).
Ocorreu ampla variação no conhecimento da associação da alimentação inadequada com as doenças e agravos não transmissíveis. Houve 80,7\% de conhecimento da relação com diabetes, enquanto apenas metade da amostra total relatou associação entre alimentação inadequada e depressão (Tabela 4).

A análise de correlação entre os escores de conhecimento é apresentada na Tabela 5. A maior correlação observada $(r=0,43)$ foi entre os conhecimentos sobre sedentarismo e alimentação inadequada. Todos os demais coeficientes variaram entre 0,30 e 0,40, exceto a relação entre os conhecimentos sobre tabagismo e consumo abusivo de álcool $(\mathrm{r}=0,22)$.

A Tabela 6 apresenta o escore médio de conhecimento para cada um dos fatores de risco conforme as variáveis independentes. As mulheres apresentaram escore de conhecimento significativamente superior ao dos homens para consumo abusivo de álcool, porém não foram observadas diferenças significativas quanto ao sexo para os outros fatores. A associação entre escores de conhecimento e idade apresentou formato de U-invertido para todos os fatores, exceto consumo abusivo de álcool; as pessoas nas faixas etárias intermediárias apresentaram os maiores escores de conhecimento. No caso do álcool, houve uma associação com tendência linear de que quanto maior a idade, maior o conhecimento sobre os malefícios do consumo abusivo. Indivíduos com pele branca apresentaram escores maiores de conhecimento, com exceção para consumo abusivo de álcool. Tanto o nível sócioeconômico quanto a escolaridade associaram-se diretamente ao conhecimento sobre sedentarismo, tabagismo e alimentação inadequada. Por 
Descrição da amostra conforme variáveis sócio-demográficas. Pelotas, Rio Grande do Sul, Brasil, 2007.

\begin{tabular}{|c|c|c|c|c|c|c|}
\hline \multirow[t]{2}{*}{ Variáveis } & \multicolumn{2}{|c|}{ Homens } & \multicolumn{2}{|c|}{ Mulheres } & \multicolumn{2}{|c|}{ Total } \\
\hline & $\mathbf{n}$ & $\%$ & $\mathrm{n}$ & $\%$ & $\mathrm{n}$ & $\%$ \\
\hline \multicolumn{7}{|l|}{ Idade (anos) } \\
\hline $10-19$ & 217 & 22,5 & 184 & 16,3 & 401 & 19,1 \\
\hline $20-29$ & 193 & 20,0 & 206 & 18,2 & 399 & 19,0 \\
\hline $30-39$ & 136 & 14,1 & 182 & 16,1 & 318 & 15,2 \\
\hline $40-49$ & 163 & 16,9 & 173 & 15,2 & 335 & 16,0 \\
\hline $50-59$ & 136 & 14,1 & 158 & 14,0 & 294 & 14,0 \\
\hline $60 \mathrm{ou}+$ & 120 & 12,4 & 229 & 20,3 & 349 & 16,7 \\
\hline \multicolumn{7}{|l|}{ Cor da pele } \\
\hline Branca & 794 & 82,5 & 924 & 81,7 & 1.718 & 82,0 \\
\hline Preta/Parda & 169 & 17,5 & 207 & 18,3 & 376 & 18,0 \\
\hline \multicolumn{7}{|l|}{ Nível sócio-econômico } \\
\hline A (mais rico) & 71 & 7,5 & 68 & 6,1 & 139 & 6,7 \\
\hline B & 326 & 34,3 & 361 & 32,4 & 687 & 33,3 \\
\hline C & 435 & 45,8 & 542 & 48,7 & 977 & 47,4 \\
\hline $\mathrm{D} / \mathrm{E}$ (mais pobres) & 118 & 12,4 & 142 & 12,8 & 260 & 12,6 \\
\hline \multicolumn{7}{|c|}{ Escolaridade (anos completos) } \\
\hline 0 & 21 & 2,2 & 68 & 6,0 & 89 & 4,3 \\
\hline $1-4$ & 205 & 21,3 & 186 & 16,5 & 391 & 18,7 \\
\hline $5-8$ & 327 & 33,9 & 362 & 32,1 & 689 & 32,9 \\
\hline $9-11$ & 249 & 25,8 & 281 & 24,9 & 530 & 25,3 \\
\hline $12 \mathrm{ou}+$ & 162 & 16,8 & 231 & 20,5 & 393 & 18,8 \\
\hline
\end{tabular}

Tabela 4

Descrição do percentual de respostas "corretas" para as associações entre fatores de risco e morbidades. Pelotas, Rio Grande do Sul, Brasil, 2007.

\begin{tabular}{lcccc}
\hline Morbidade & & \multicolumn{2}{c}{ Respostas corretas (\%) } \\
& Sedentarismo & Tabagismo & Álcool & Alimentação \\
\hline Diabetes & 50,9 & 23,8 & 48,9 & 80,7 \\
Hipertensão arterial & 70,6 & 77,2 & 82,9 & 76,8 \\
AIDS & 90,5 & 89,6 & 5,2 & 90,8 \\
Osteoporose & 63,7 & 39,2 & 37,6 & 77,8 \\
Câncer de pulmão & 16,7 & 96,9 & 31,3 & 24,4 \\
Depressão & 70,7 & 37,6 & 76,5 & 52,2 \\
Cirrose hepática & 81,5 & 61,0 & 92,4 & 57,8 \\
Infarto agudo do miocárdio & 81,4 & 89,4 & 79,6 & 68,9 \\
\hline
\end{tabular}

outro lado, houve associação inversa com consumo abusivo de álcool.

Realizou-se análise ajustada por meio de regressão linear múltipla para avaliar o efeito das variáveis sexo, idade, cor da pele, nível sócioeconômico e escolaridade sobre os escores de conhecimento com ajuste para os potenciais fatores de confusão. A maior parte das associações foi consistente com os dados da análise bruta, com exceção da variável cor da pele, que perdeu a associação com os escores de tabagismo e alimentação inadequada. Considerando-se a similaridade entre os achados das análises brutas e os das ajustadas, optou-se por apresentar apenas os primeiros em tabelas. 
Análise de correlação (Spearman) entre os escores de conhecimento para cada um dos fatores de risco. Pelotas, Rio Grande do Sul, Brasil, 2007.

\begin{tabular}{lccc}
\hline Sedentarismo & Tabagismo & $\begin{array}{c}\text { Consumo excessivo } \\
\text { de álcool }\end{array}$ & $\begin{array}{c}\text { Alimentação } \\
\text { inadequada }\end{array}$ \\
\hline $\begin{array}{l}\text { Sedentarismo } \\
\text { Tabagismo }\end{array}$ & $r s=0,32$ & & \\
Consumo excessivo de álcool & $p<0,001$ & & \\
& $r s=0,37$ & $r s=0,22$ & \\
Alimentação inadequada & $p<0,001$ & $p<0,001$ & \\
& $r s=0,43$ & $r s=0,31$ & $r s=0,39$ \\
& $p<0,001$ & $p<0,001$ & $p<0,001$ \\
\hline
\end{tabular}

Tabela 6

Escores médios de conhecimento dos fatores de risco conforme variáveis sócio-demográficas. Pelotas, Rio Grande do Sul, Brasil, 2007.

\begin{tabular}{|c|c|c|c|c|c|c|c|c|}
\hline \multirow[t]{2}{*}{ Variáveis } & \multicolumn{2}{|c|}{ Sedentarismo } & \multicolumn{2}{|c|}{ Tabagismo } & \multicolumn{2}{|c|}{ Álcool } & \multicolumn{2}{|c|}{ Alimentação } \\
\hline & Média & DP & Média & DP & Média & DP & Média & DP \\
\hline Sexo * & \multicolumn{2}{|c|}{$p=0,63$} & \multicolumn{2}{|c|}{$p=0,1$} & \multicolumn{2}{|c|}{$p=0,005$} & \multicolumn{2}{|c|}{$p=0,13$} \\
\hline Masculino & 4,7 & 1,7 & 5,2 & 1,3 & 4,4 & 1,8 & 5,2 & 1,6 \\
\hline Feminino & 4,7 & 1,6 & 5,1 & 1,3 & 4,6 & 1,7 & 5,3 & 1,5 \\
\hline Idade (anos) ** & \multicolumn{2}{|c|}{$p<0,001 * \star \star$} & \multicolumn{2}{|c|}{$p<0,001 * \star \star$} & \multicolumn{2}{|c|}{$\mathrm{p}<0,001$ *** } & \multicolumn{2}{|c|}{$\mathrm{p}<0,001 * \star \star$} \\
\hline $10-19$ & 4,2 & 1,8 & 4,7 & 1,5 & 3,7 & 2,0 & 4,6 & 1,8 \\
\hline $20-29$ & 4,9 & 1,4 & 5,3 & 1,1 & 4,3 & 1,5 & 5,7 & 1,2 \\
\hline $30-39$ & 4,9 & 1,4 & 5,3 & 1,0 & 4,6 & 1,4 & 5,7 & 1,3 \\
\hline $40-49$ & 5,0 & 1,6 & 5,4 & 1,1 & 4,9 & 1,5 & 5,5 & 1,4 \\
\hline $50-59$ & 4,8 & 1,7 & 5,2 & 1,3 & 4,9 & 1,7 & 5,3 & 1,6 \\
\hline $60 \mathrm{ou}+$ & 4,5 & 1,8 & 5,0 & 1,3 & 5,1 & 1,8 & 5,1 & 1,6 \\
\hline Cor da pele * & \multicolumn{2}{|c|}{$p<0,001$} & \multicolumn{2}{|c|}{$p<0,001$} & \multicolumn{2}{|c|}{$p=0,87$} & \multicolumn{2}{|c|}{$p<0,001$} \\
\hline Branca & 4,8 & 1,6 & 5,2 & 1,2 & 4,5 & 1,7 & 5,4 & 1,5 \\
\hline Preta/Parda & 4,4 & 1,9 & 4,9 & 1,4 & 4,5 & 2,0 & 5,0 & 1,7 \\
\hline Nível sócio-econômico ** & \multicolumn{2}{|c|}{$\mathrm{p}<0,001 \star \star \star *$} & \multicolumn{2}{|c|}{$\mathrm{p}<0,001 \star \star \star *$} & \multicolumn{2}{|c|}{$p=0,02$} & \multicolumn{2}{|c|}{ 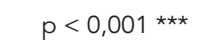 } \\
\hline A (mais ricos) & 5,0 & 1,3 & 5,6 & 1,0 & 4,3 & 1,5 & 5,6 & 1,2 \\
\hline B & 4,8 & 1,6 & 5,2 & 1,2 & 4,5 & 1,6 & 5,5 & 1,5 \\
\hline C & 4,7 & 1,7 & 5,1 & 1,3 & 4,6 & 1,8 & 5,2 & 1,6 \\
\hline D/E (mais pobres) & 4,3 & 1,8 & 4,9 & 1,4 & 4,8 & 1,9 & 5,0 & 1,7 \\
\hline Escolaridade (anos) $\star \star$ & \multicolumn{2}{|c|}{$p<0,001 * \star \star$} & \multicolumn{2}{|c|}{$p<0,001 * \star \star *$} & \multicolumn{2}{|c|}{$p=0,01 \star \star \star$} & \multicolumn{2}{|c|}{$\mathrm{p}<0,001 * \star \star *$} \\
\hline 0 & 3,5 & 2,1 & 4,4 & 1,6 & 5,0 & 2,3 & 4,6 & 1,8 \\
\hline $1-4$ & 4,2 & 1,9 & 4,7 & 1,6 & 4,6 & 2,1 & 4,6 & 1,9 \\
\hline $5-8$ & 4,7 & 1,6 & 5,1 & 1,2 & 4,6 & 1,7 & 5,2 & 1,5 \\
\hline 9-11 & 5,0 & 1,4 & 5,4 & 1,1 & 4,5 & 1,6 & 5,6 & 1,3 \\
\hline 12 ou mais & 5,1 & 1,4 & 5,5 & 1,0 & 4,4 & 1,4 & 5,9 & 1,0 \\
\hline
\end{tabular}

DP: desvio-padrão.

* Teste t;

** Análise de variância;

$\star \star \star$ Teste de Kruskal-Wallis. 


\section{Discussão}

O presente estudo encontrou alto percentual de respostas corretas para as associações mais difundidas na literatura científica. Mais de $80 \%$ dos entrevistados mostraram-se cientes das associações entre sedentarismo e infarto agudo do miocárdio; tabagismo e câncer de pulmão; consumo abusivo de álcool e cirrose; alimentação inadequada e diabetes. Associações inexistentes também foram corretamente identificadas por uma grande parcela dos respondentes, como, por exemplo, a ausência de associação dos fatores sedentarismo, tabagismo e alimentação inadequada com AIDS.

O principal desafio metodológico do presente estudo foi a classificação de algumas associações como existentes ou não, gerando escores de respostas "corretas". Algumas das associações investigadas são amplamente relatadas na literatura com consistência entre os diversos estudos, por exemplo, tabagismo e infarto agudo do miocárdio. Outras são menos estudadas ou os resultados são menos consistentes, como sedentarismo e câncer de pulmão. Optamos por apresentar (Tabela 2) os estudos que nortearam nossas decisões quanto ao "certo" e "errado", de forma que leitores possam identificar a fonte de nossas decisões, as quais foram baseadas em revisões sistemáticas ou meta-análises. Sabe-se que tais decisões podem suscitar discordâncias. A escolha de "certo" e "errado" foi arbitrária, mas fundamental para fins de análise, e não deve ser compreendida como uma verdade científica definitiva. Não se espera que as pessoas tenham o mesmo conhecimento sobre associações consagradas na literatura e outras que vêm recebendo atenção apenas nos últimos anos. Outra limitação do estudo envolve os conceitos de alimentação "adequada" e consumo "exagerado" de álcool. Os respondentes não receberam qualquer orientação sobre o critério para definir uma dieta como adequada ou não ou o consumo de álcool como exagerado ou não. Optou-se por utilizar apenas a percepção dos entrevistados.

Outro aspecto metodológico importante é a utilização de um instrumento criado pelos próprios pesquisadores. Em primeiro lugar, deve-se deixar claro que não existe um padrão-ouro para avaliação de conhecimento populacional sobre fatores de risco para doenças e agravos não transmissíveis. Desta forma, optamos por utilizar um instrumento padronizado e com questões idênticas para os quatro fatores de risco, permitindo análises comparativas. Para cada um dos fatores, existia um número variado de respostas positivas corretas, indo de cinco (tabagismo) a oito (consumo abusivo de álcool).
Estudos populacionais que investiguem conhecimento de fatores de risco para doenças crônicas são raros. A maioria das pesquisas sobre esse tema tratam de doenças infecto-contagiosas, especialmente AIDS 4,5. Uma questão metodológica importante é que vários estudos tratam do conhecimento que sujeitos já acometidos pelas doenças têm ${ }^{6,7}$. Em nosso caso, os indivíduos foram escolhidos aleatoriamente na população geral, minimizando a possibilidade de viés de seleção.

A ausência de conhecimento populacional sobre algumas associações merece atenção especial. Cerca de metade dos sujeitos e dois terços deles, respectivamente, estão cientes da associação do sedentarismo com diabetes mellitus e osteoporose. Tais relações causais são extremamente consistentes na literatura $8,9,10,11$, mas claramente esse conhecimento não foi incorporado pela população em geral. Igualmente, menos de $40 \%$ dos entrevistados mostraram-se cientes da associação entre tabagismo e osteoporose, a qual também é bastante clara na literatura científica 12. O consumo abusivo de álcool não foi identificado pela maioria dos respondentes como fator de risco para diabetes (49\%), câncer de pulmão (31\%) e osteoporose (38\%). Uma possível explicação para esse achado é a abundante quantidade de informações referentes a cirrose e acidentes de trânsito como malefícios do álcool, sendo os outros agravos menos divulgados na mídia. Notou-se também baixo conhecimento populacional sobre a relação existente entre alimentação inadequada, sedentarismo e câncer de pulmão, o que pode ser explicado pelo fato de a população reconhecer claramente o tabagismo como principal fator de risco para câncer de pulmão.

Os resultados para as correlações entre os escores de conhecimento foram consistentemente moderados ou baixos, parecendo haver certa independência entre eles. A maior correlação encontrada entre os conhecimentos de sedentarismo e alimentação inadequada pode ser reflexo de campanhas governamentais e midiáticas 13 , as quais ressaltam, na maioria das vezes, a importância conjunta da prática de atividade física regular e alimentação adequada para a saúde. Por outro lado, as campanhas contra tabagismo ou consumo de álcool normalmente são realizadas de forma isolada.

Apesar de um estudo anterior ter encontrado maiores escores de conhecimento para mulheres 2 , nossos achados não confirmaram essa tendência. Apenas para o consumo excessivo de álcool, as mulheres tiveram maior nível de conhecimento do que os homens, sendo a magnitude dessa diferença bastante reduzida. 
Os escores de conhecimento, em geral, foram superiores nos estratos sociais mais favorecidos da população, seja quanto à escolaridade, seja quanto ao nível sócio-econômico. É função da saúde pública compreender de que forma essa distinção social ocorre, podendo ser um efeito direto da falta de escolarização, um menor acesso a informações de qualidade nos meios de comunicação, ou piores cuidados em saúde. Independentemente da causa, o que se pode notar é que, em algum momento, esses sistemas falharam, ajudando a agravar a desigualdade sócio-econômica observada no país. Diferentemente do observado para os outros fatores, o conhecimento sobre os malefícios do consumo abusivo de álcool foi maior nos grupos sociais mais desfavorecidos, porém tal achado é de difícil explicação e estudos futuros podem aprofundar essa temática.

Apesar da relevância do conhecimento, e da relação existente entre conhecimento e com-

\section{Resumo}

O objetivo deste estudo foi avaliar o conhecimento populacional sobre as associações de quatro fatores comportamentais (sedentarismo, tabagismo, consumo excessivo de álcool e alimentação inadequada) com oito morbidades (diabetes, hipertensão arterial, AIDS, osteoporose, câncer de pulmão, depressão, cirrose hepática e infarto agudo do miocárdio). Foi realizado um estudo transversal de base populacional, incluindo 2.096 indivíduos de dez anos ou mais. O processo de amostragem foi probabilístico por conglomerados. Para cada fator comportamental, foi gerado um escore de conhecimento, que variava de zero a oito pontos. A maior média do escore foi registrada para o conhecimento sobre alimentação inadequada $(5,3)$, seguida por tabagismo $(5,1)$, sedentarismo $(4,7)$ e consumo excessivo de álcool $(4,5)$. Em geral, maiores escores de conhecimento estiveram relacionados com maior es colaridade, nível sócio-econômico e faixas etárias intermediárias. Estratégias governamentais a fim de aumentar o conhecimento populacional sobre fatores de risco para doenças crônicas são necessárias. portamento 2, estudos têm mostrado que o conhecimento isoladamente não é suficiente para promover modificações no comportamento das pessoas 14 . Esse paradigma de dualidade entre conhecimento e mudança de comportamento apresenta-se como desafiador para a saúde pública, visto que alarmantes prevalências de sedentarismo, tabagismo, consumo abusivo de álcool e obesidade são observadas exatamente em um momento no qual o conhecimento populacional é relativamente elevado. Entendemos, no entanto, que o conhecimento, embora não suficiente para mudança de comportamento, seja fundamental, além de ser um passo importante em direção a uma sociedade mais saudável. Estratégias governamentais de promoção da saúde são necessárias, visando a aumentar o conhecimento sobre fatores de risco para doenças e agravos não transmissíveis e, como conseqüência, modificar o estilo de vida da população brasileira.

\section{Colaboradores}

T. T. Borges liderou a escrita do artigo e a análise de dados. A. J. Rombaldi e P. C. Hallal atuaram na concepção, análise dos dados e na redação do artigo. A. G. Knuth auxiliou na coleta de dados, análise e escrita do manuscrito.

\section{Agradecimentos}

P. C. Hallal é bolsista de produtividade do Conselho Nacional de Desenvolvimento Científico e Tecnológico (CNPq). 


\section{Referências}

1. Vuori I, Paronen O, Oja P. How to develop local physical activity promotion programmes with national support: the Finnish experience. Patient Educ Couns 1998; 33(1 Suppl):S111-9.

2. Domingues MR, Araújo CLP, Gigante DP. Conhecimento e percepção sobre exercício físico em uma população adulta urbana do Sul do Brasil. Cad Saúde Pública 2004; 20:204-15.

3. Knuth AG, Bielemann RM, Silva SG, Borges TT, Del Duca GF, Kremer MM, et al. Conhecimento de adultos sobre o papel da atividade física na prevenção e tratamento de diabetes e hipertensão: estudo de base populacional no Sul do Brasil. Cad Saúde Pública 2009; 25:513-20.

4. Ferreira MP. Conhecimento e percepção de risco sobre o HIV/AIDS: um perfil da população brasileira no ano de 1998. Cad Saúde Pública 2003; 19 Suppl 2:S213-22.

5. Martins LBM, Costa-Paiva LHS, Osis MJD, Sousa MH, Pinto-Neto AM, Tadini V. Fatores associados ao uso de preservativo masculino e ao conhecimento sobre DST/AIDS em adolescentes de escolas públicas e privadas do Município de São Paulo, Brasil. Cad Saúde Pública 2006; 22:315-23.

6. Sabri AA, Qayyum MA, Saigol NU, Zafar K, Aslam F. Comparing knowledge of diabetes mellitus among rural and urban diabetics. McGill J Med 2007; 10:87-9.

7. Viera AJ, Cohen LW, Mitchell CM, Sloane PD. High blood pressure knowledge among primary care patients with known hypertension: a North Carolina Family Medicine Research Network (NC-FM-RN) study. J Am Board Fam Med 2008; 21:300-8.

8. Bauman AE. Updating the evidence that physical activity is good for health: an epidemiological review 2000-2003. J Sci Med Sport 2004; 7:6-19.

9. Pitanga FJ. Epidemiologia, atividade física e saúde. Rev Bras Ciênc Mov 2002; 10:49-54.

10. Sartorelli DS, Franco LJ. Tendências do diabetes mellitus no Brasil: o papel da transição nutricional. Cad Saúde Pública 2003; 19 Suppl 1:S29-36.

11. Pols MA, Peeters PH, Kemper HC, Grobbee DE. Methodological aspects of physical activity assessment in epidemiological studies. Eur J Epidemiol 1998; 14:63-70.

12. Spangler JG, Quandt S, Bell RA. Smokeless tobacco and osteoporosis: a new relationship? Med Hypotheses 2001; 56:553-7.

13. World Health Organization. Global strategy on diet, physical activity and health. Geneva: World Health Organization; 2004.

14. Menezes A, Assunção MC, Neutzling MB, Malcon M, Hallal PC, Marques A, et al. Effectiveness of an educational intervention on smoking, diet and physical activity among adolescents. Pelotas: World Health Organization/Instituto Nacional de Câncer; 2008.

15. Bassuk SS, Manson JE. Epidemiological evidence for the role of physical activity in reducing risk of type 2 diabetes and cardiovascular disease. J Appl Physiol 2005; 99:1193-204.
16. American College of Sports Medicine. Position Stand. Physical activity, physical fitness, and hypertension. Med Sci Sports Exerc 1993; 25:i-x.

17. Sinaki M. Exercise and osteoporosis. Arch Phys Med Rehabil 1989; 70:220-9.

18. Tardon A, Lee WJ, Delgado-Rodriguez M, Doseme ci M, Albanes D, Hoover R, et al. Leisure-time physical activity and lung cancer: a meta-analysis. Cancer Causes Control 2005; 16:389-97.

19. Teychenne M, Ball K, Salmon J. Physical activity and likelihood of depression in adults: a review. Prev Med 2008; 46:397-411.

20. Sofi F, Capalbo A, Cesari F, Abbate R, Gensini GF. Physical activity during leisure time and primary prevention of coronary heart disease: an updated meta-analysis of cohort studies. Eur J Cardiovasc Prev Rehabil 2008; 15:247-57.

21. Will JC, Galuska DA, Ford ES, Mokdad A, Calle EE. Cigarette smoking and diabetes mellitus: evidence of a positive association from a large prospective cohort study. Int J Epidemiol 2001; 30:540-6.

22. Westman EC. Does smokeless tobacco cause hypertension? South Med J 1995; 88:716-20.

23. Høidrup S, Prescott E, Sørensen T, Gottschau A, Lauritzen JB, Schroll M, et al. Tobacco smoking and risk of hip fracture in men and women. Int J Epidemiol 2000; 29:253-9.

24. Hackshaw AK, Law MR, Wald NJ. The accumulated evidence on lung cancer and environmental tobacco smoke. BMJ 1997; 315:980-8.

25. Critchley JA, Capewell S. Mortality risk reduction associated with smoking cessation in patients with coronary heart disease: a systematic review. JAMA 2003; 290:86-97.

26. Howard AA, Arnsten JH, Gourevitch MN. Effect of alcohol consumption on diabetes mellitus: a systematic review. Ann Intern Med 2004; 140:211-9.

27. Beilin LJ, Puddey IB. Alcohol and hypertension: an update. Hypertension 2006; 47:1035-8.

28. Bryant KJ. Expanding research on the role of alcohol consumption and related risks in the prevention and treatment of HIV/AIDS. Subst Use Misuse 2006; 41:1465-507.

29. Bikle DD. Effects of alcohol abuse on bone. Compr Ther 1988; 14:16-20.

30. Chao C. Associations between beer, wine, and liquor consumption and lung cancer risk: a metaanalysis. Cancer Epidemiol Biomarkers Prev 2007; 16:2436-47.

31. Madden JS. Alcohol and depression. Br J Hosp Med 1993; 50:261-4.

32. Corrao G, Bagnardi V, Zambon A, Torchio P. Metaanalysis of alcohol intake in relation to risk of liver cirrhosis. Alcohol Alcohol 1998; 33:381-92.

33. Biyik I, Ergene O. Alcohol and acute myocardial infarction. J Int Med Res 2007; 35:46-51.

34. Steyn NP, Mann J, Bennett PH, Temple N, Zimmet P, Tuomilehto J, et al. Diet, nutrition and the prevention of type 2 diabetes. Public Health Nutr 2004; 7:147-65. 
35. Srinath Reddy K, Katan MB. Diet, nutrition and the prevention of hypertension and cardiovascular diseases. Public Health Nutr 2004; 7:167-86.

36. Atkinson SA, Ward WE. Clinical nutrition: 2 . The role of nutrition in the prevention and treatment of adult osteoporosis. CMAJ 2001; 165:1511-4.
37. Ziegler RG, Mayne ST, Swanson CA. Nutrition and lung cancer. Cancer Causes Control 1996; 7:157-77.

38. Alpert JE, Fava M. Nutrition and depression: the role of folate. Nutr Rev 1997; 55:145-9.

39. Hu FB, Willett WC. Optimal diets for prevention of coronary heart disease. JAMA 2002; 288:2569-78.

Recebido em 10/Dez/2008

Versão final reapresentada em 03/Fev/2009

Aprovado em 06/Abr/2009 